\title{
DESIGN AND PERFORMANCE ANALYSIS OF SEMICONDUCTOR OPTICAL AMPLIFIER FOR 16×10Gbps DWDM TRANSMISSION SYSTEMS
}

\author{
Bhavesh Ahuja, M.L. Meena and R.S. Meena \\ Department of Electronics Engineering, Rajasthan Technical University, India
}

\begin{abstract}
In this paper, we proposed sixteen channel dense-wavelength-divisionmultiplexing (DWDM) optical transmission system using semiconductor optical amplifiers (SOAs). The SOA amplifiers overcome the attenuation effects of transmitted signal in optical communication systems. To overcome attenuation effects, pre-, post (booster) and In-line SOAs techniques are modeled, analyzed and compared for investigating the performance of DWDM system. The proposed system is designed for 10Gbps network using non-return-tozero (NRZ) modulation format with dispersion compensation fiber (DCF) of length 14kms and a single mode fiber (SMF) of length $70 \mathrm{kms}$ using Optisystem7.0 simulator. Performance of designed system is explored and compared in terms of eye shape (eye-diagram), $Q$-factor and bit error rate (BER) by varying the input power ( $\mathrm{mw}$ ) parameter of $C W$ laser source. It is observed that the In-line SOA amplifier provides the best performance as attenuation compensator for proposed optical transmission systems.
\end{abstract}

Keywords:

Bit Error Rate, Dense Wavelength Division Multiplexing, Dispersion Compensation Fiber, In-Line Amplifier, Semiconductor Optical Amplifier

\section{INTRODUCTION}

Nowadays, the demand for high speed data transfer network is increasing day by day due to fast expansion in the number of international or global users of internet. Dense-wavelengthdivision-multiplexing (DWDM) system is currently been adopted to increase the data carrying ability and an efficient utilization of optical networks for long-haul optical communication systems [1] [2]. DWDM is a method of transmission system that uses multiplexer at transmitter division for accumulating and transmitting different wavelength light signals onto a single channel and de-multiplexer at receiver division to spread the light signals from the channel to the respective channel from a single fiber as can be seen in Fig.1.

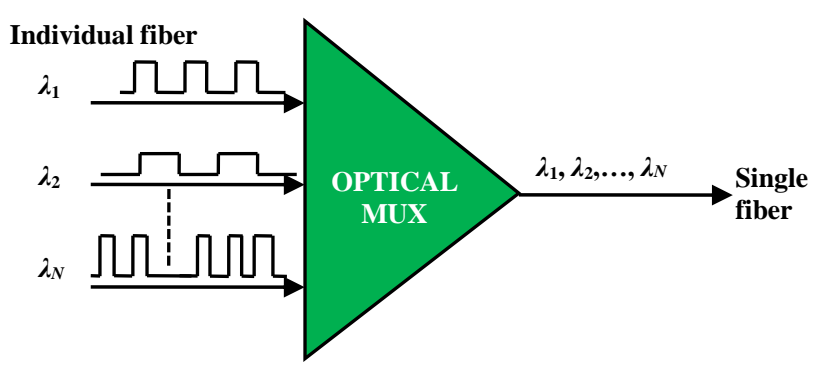

Fig.1. Basic building block of optical DWDM transmission system

The long distance transmission in optical DWDM systems is mainly restricted by fiber non-linearity, dispersion and attenuation. To overcome dispersion, lots of techniques are suggested such as Dispersion Compensation Fiber (DCF), Dispersion Compensation Filter or higher order mode filters and Fiber Bragg Grating (FBG) [3] [4]. Among all these dispersion justifying techniques, the FBG is the most efficient and cost effective dispersion compensation technique [5]. Further, optical signal attenuation compensates in two manners: by conventional optoelectronic repeaters and optical amplifiers (OAs). In optoelectronic repeaters the optical signal is converted into electrical signal (O-E) and thus regenerated by transmitter as can be seen in Fig. 2 .

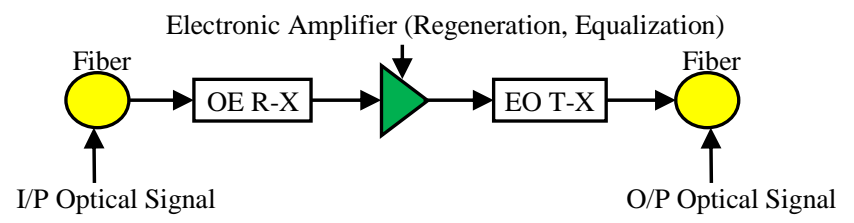

Fig.2. Basic principal of optical communication systems

However, the regenerators are relatively complex, more time consuming and expensive for DWDM light wave systems because it requires many processing stages, multiplexing, opticalelectrical-optical (O-E-O) conversion, and de-multiplexing. An alternative approach is to manage the attenuation by optical amplifiers, which are directly amplifying the transmitted optical signals of different wavelength simultaneously without the domain conversions [6] [7]. The basic concepts of an optical amplifier (OAs) are based on principle of conventional laser. In which we raise or increases optical signal power by pumping the power in an optical amplifier medium as can be seen in Fig.3. For long-haul transmission, optical amplifiers are most efficient, stable with minimum attenuations and it is essential to maintain the required level of systems performance in terms of larger gain bandwidth, gain flatness, power crosstalk and transient performance for DWDM systems [8].

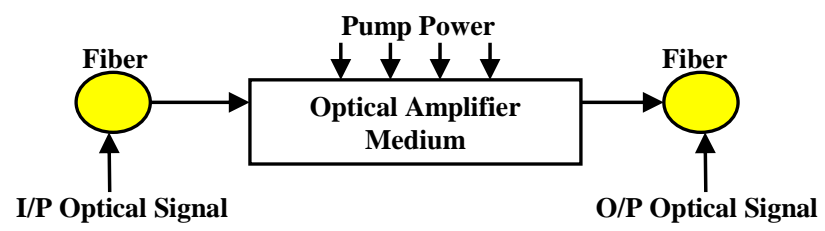

Fig.3. Basic principal of optical amplifiers (OAs)

\section{OPTICAL AMPLIFIERS}

Presently, Erbium Doped Fiber Amplifier (EDFA), Raman Amplifier (RA) and Semiconductor Optical Amplifier (SOA) are generally used in DWDM systems to overcome the attenuation phenomena. 


\subsection{ERBIUM DOPED FIBER AMPLIFIER}

The EDFA is a short length segment of fiber which is doped with erbium ions. These erbium ions are excited by pumped laser source at numerous optical frequencies (to higher energy level). The most suitable excitation wavelengths are $980 \mathrm{~nm}$ and $1480 \mathrm{~nm}$. While these wavelengths transmit through a fiber, erbium ions are energized and an input optical signal can be amplified by stimulated emission and release photon energy in the wavelength band of $1530-1565 \mathrm{~nm}$. Therefore, according to modify the amplifier design, wavelength band can be shifted to longer wavelengths. The basic configuration of an EDFA can be seen in Fig.4 which consists coupler, erbium doped fiber and two isolators. Fiber carrying the input signal via isolator that suppresses optical reflections and stimulated through high pump laser power at $980 \mathrm{~nm}$ or $1480 \mathrm{~nm}$ is coupled into EDFA together with input signal. The pump laser excites fiber doping ions and signal amplifies in wavelength region of $1550 \mathrm{~nm}$. The EDFA is used as booster and in-line amplifier for long distance transmission. Also amplifies multi-channel signals on different wavelengths simultaneously with low noise figure. But the major shortcoming of EDFAs is wavelength dependent low gain bandwidth spectrum 40nm occurs during the stimulated emission [9].

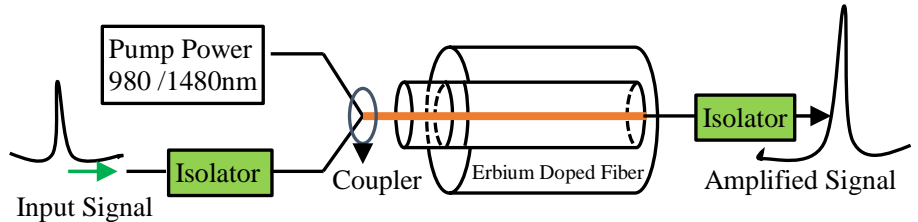

Fig.4. Basic principal of EDFA amplifier

\subsection{RAMAN AMPLIFIER}

Raman amplifier is an optical amplification mechanism, in which signal is amplified by Stimulated Raman Scattering (SRS). To achieve stimulated scattering, light is scattered through atoms from a lower to higher wavelength. In SRS, powerful pump beam is propagating through silica fiber for arises gain by transferring the power from shorter wavelength to longer wavelength (known as down shift in frequency or Stokes shift) [10]. Therefore, Raman scattering is a nonlinear interaction between pump wavelength (lower) and signal wavelength (higher) in any optical fiber (both signal and pump wavelength are different). The basic concept of fiber-based Raman amplifier can be seen in Fig.5.

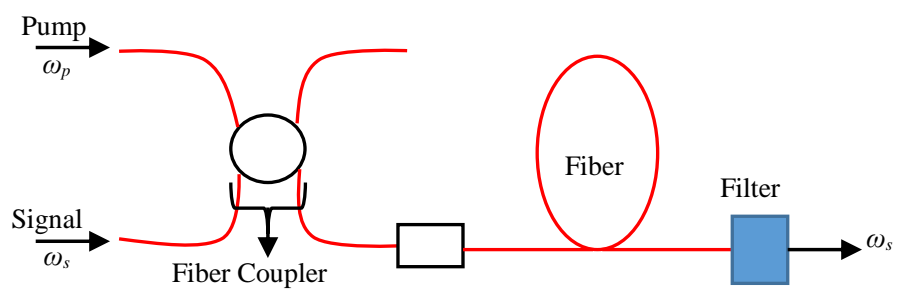

Fig.5. Basic principal of fiber-based Raman amplifier

The advantage of RAs are low noise figure and wide gain spectrum (up to $10 \mathrm{~nm}$ ), it can be changed by varying the number of pumps and pump wavelengths. Further, Raman amplifier requires high pump power for batter efficiency and expensive high power lasers, for delivering high power into single mode fiber [11]. Two types of RAs are basically used on their design knows as: Distributed Raman amplifier (DRA), in which longer transmission fiber (approx. 100km) is used as medium gain by combining pump wavelength with signal wavelength. Another is Discrete Raman amplifier, in which shorter length of fiber (approx. 20km) is used for amplification. The main motive of reduced fiber span is highly non-linear with small core is used to increase the interaction between signal and pump wavelengths.

\subsection{SEMICONDUCTOR OPTICAL AMPLIFIER}

In semiconductor amplifiers (SOAs) energy is applied via as pump or electrical current to attain population inversion. The schematic of semiconductor optical amplifier is shown in Fig.6. Accordingly, the input signal is amplified through entire active area as a result of coherent semiconductor stimulated emission. The basic principle of spontaneous and stimulated process can be seen in Fig.7. Therefore, the signal amplification through SOA is based on recombination of electrons and holes pairs of highly doped $p$ - $n$ type semiconductor structure. The SOAs are made as chip in an enclosed cavity to keep constant regulating temperature and appropriate wavelength to acquire maximum gain. Hence, SOAs are similar to lasers cavity but difference is that SOA amplifier include anti-reflex layer against formation of resonance and signal gathering inside medium. In SOAs, good quantum efficient semiconductor materials (GaAs, Al-Ga-AS, In-Ga-As, In-Ga-AsP, and InP) are used during manufacturing.

In the medium of amplification process, some noise can be accompanied with amplified output signal which is known as amplified spontaneous emission (ASE) noise, which belongs to limit the transmission distance. Therefore, the main problems in SOAs are largest amount of ASE and their gain dynamics can cause serious signal distortion. In order to overcome this problem, the impulsive or spontaneous carrier-lifetime in active region of material is set at much smaller time period than other amplifiers. Due to faster carrier recombination life-time, the amplifier gain is dynamics because amplifier will react quickly according to the changes in input optical signal power. It may cause signal distortions in multi-channel transmission systems because optical signals are amplified all together [12].

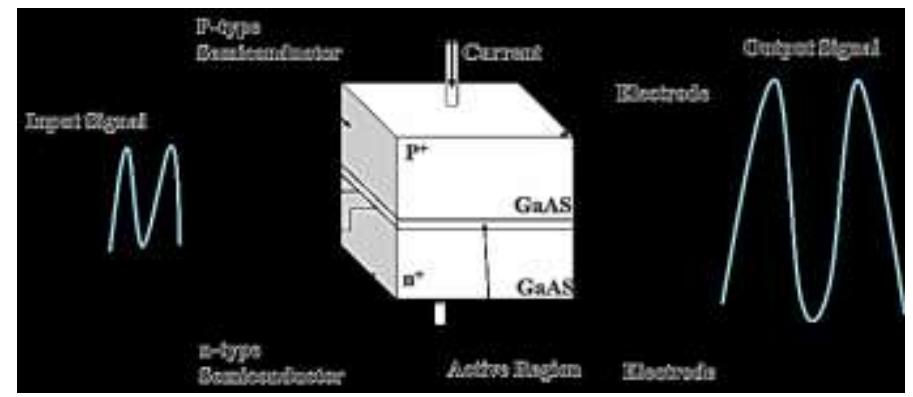

Fig.6: Basic schematics of semiconductor optical amplifier

The 100Gbps DWDM system is simulated via cascaded SOAs for 10 channels with DPSK modulation format, it is found that low saturation power $21.36 \mathrm{~mW}$, low crosstalk $14 \mathrm{~dB}$, and high optical gain $36 \mathrm{~dB}$ is achieved with optimized SOAs. Further, for $70 \mathrm{~km}$ transmission distance, the achieved output signal power via optimized SOA amplifier at same quality without in-line amplifier. Therefore, using optimum span scheme it is possible to transmit $100 \mathrm{Gbps}$ RZ-DPSK signal at $17,227 \mathrm{~km}$ with power penalty of $2 \mathrm{~dB}$ good quality of signal [13]. The post-power 
amplifier compensation method shows good performance in terms of bit-error-rate (BER), eye-closure penalty and received power as compared to pre and symmetrical power compensation amplifier methods. Therefore, the BER and eye-closure penalty increases with increase in signal input power [14]. Further, the semiconductor amplifier gives amplification in range of 1310$550 \mathrm{~nm}$ to facilitate broad amplification band-width however its operation is restricted to $10 \mathrm{Gbps}$, polarization dependent, large noise figure, cross talk, lower gain and large signal distortion. Thus, SOAs used for small optical networks [9]. The semiconductor optical amplifier gives improved performance at small dispersion $(D=2 \mathrm{ps} / \mathrm{nm} / \mathrm{km})$ and less distance; when less number of channels is used in WDM systems and performance will degrades by increasing the number of channels [15], [16], [19], [20].

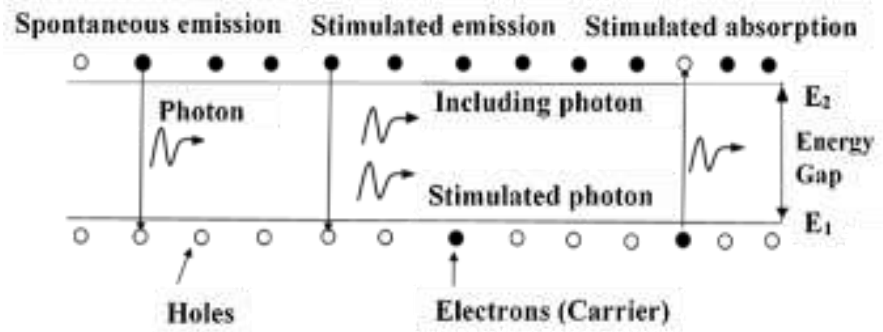

Fig.7. Basic principal of spontaneous and stimulated emission process

It is concluded from literature, the fundamental theory of all optical amplifiers is nearly identical but its continuous existence in particular application depends on features and constraints. Therefore, from the study of literature, the semiconductor optical amplifiers (SOAs) are best suitable for fast chip switching for routing in WDM systems, logic gates and wavelength converters because of wide amplification band-width (approx.70nm), compact size, low cost and less power compensation as compared to Raman and EDFA [17]. Further, the EDFA and Raman amplifier makes transmission system costly as it will require highpump power to operate, whereas SOAs need only an electrical current at level of around 50mA [18]. Thus, due to the encroachment in optical semiconductor fabrication techniques, the design of SOAs is an enormous promise for developing optical transmission systems. The semiconductor optical amplifiers can be utilized in optical switching, wavelength conversion and as gain element. In this work we present the SOA technology and their applications in promising optical transmission systems.

The proposed work presents the performance of DWDM transmission system through In-line semiconductor optical amplifier for $16 \times 10 \mathrm{Gbps}$ optical link. The optimized parameters of SMF as well as SOAs are identified and after simulation, performance parameters of the proposed model are analyzed, tested and compared with standard pre- and booster SOAs schemes in terms of quality of eye shape, BER and Q-factor by varying input power $\mathrm{CW}$ laser source. Then, we also compared the proposed model with previously reported optical system by researchers with SOAs as attenuation compensator [5] [9] [13] [14]. Finally, it can be observed that, the reported work improves the performance of proposed model by dropping attenuation phenomena through In-line SOA amplifier. The designed optical DWDM configurations are modeled and simulated by OptiSystem7.0 simulator.

\section{PROPOSED SYSTEM MODEL}

The 16-channels DWDM optical transmission system is designed for evaluating the performance of semiconductor optical amplifiers (SOAs) based on pre, post (booster), and in-line amplifier topologies. The proposed model is simulated by Optisystem7.0 simulator by varying input power to investigate how different amplifier topologies can alter the performance of DWDM transmission systems at $10 \mathrm{Gbps}$ per channel data speed. The fundamental block diagram of proposed system is shown in Fig.8. The proposed optical systems can be divided into main categories which are; transmitter section, channel (media) and receiver section. The transmitter section contains four basic blocks (data source, electrical driver (pulse generator), CW laser source and Mach-Zehnder modulator). In each channel the transmitter is constructed by data source, to produce a pseudo random bit sequence (PRBS) at the bit rate of $10 \mathrm{Gbps}$ from where a binary signal is transmitted, non-return-to-zero (NRZ) pulse generator, to convert binary data into electrical pulse, continuous wave laser $(\mathrm{CW})$ and Mach-Zehnder modulator (MZM) to modulate the $\mathrm{CW}$ laser signal. The Mach-Zehnder modulator receives electrical signal as well as optical signal and induces phase shift between two signals if needed and thus providing an optical signal at output. The insertion loss, chirp factor and extinction ratio of MZ-modulator is selected to be $5 \mathrm{~dB}, 0.5$ and $30 \mathrm{~dB}$, respectively. The central frequencies of each channel (for 16-channel simulation-starting from $1^{\text {st }}$ to $16^{\text {th }}$ channel) are selected from $193.1 \mathrm{THz}-194.6 \mathrm{THz}$ according to recommendation of ITU-TG.694.1.

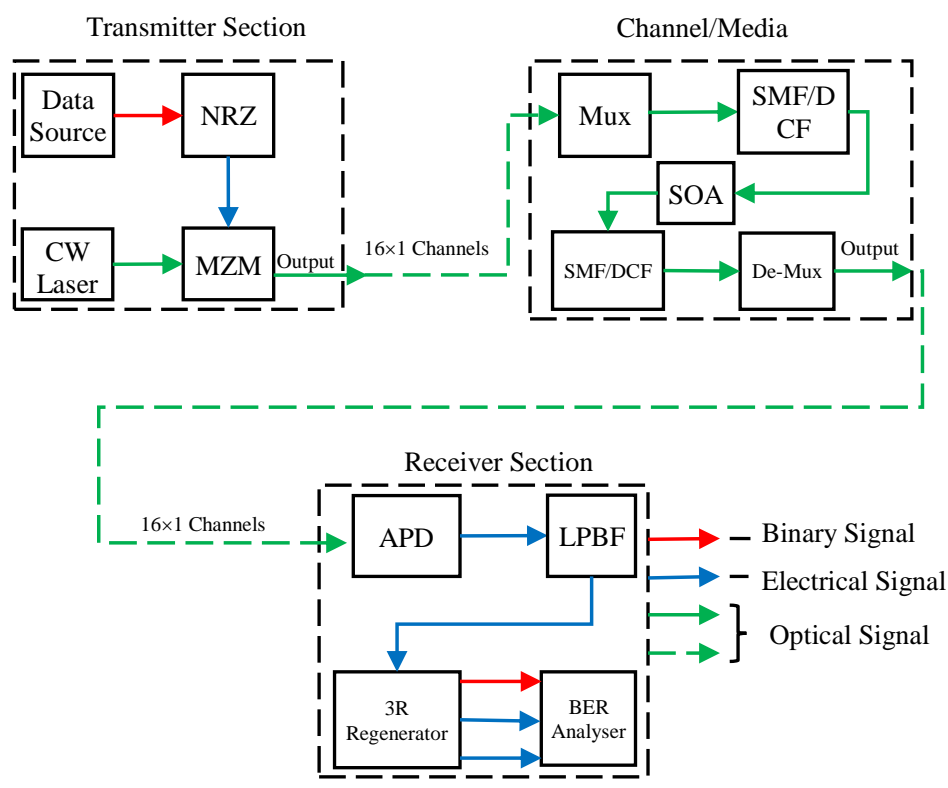

Fig.8. Building blocks of proposed 16-channel DWDM optical transmission system for evaluate the performance of SOA amplifier topologies 


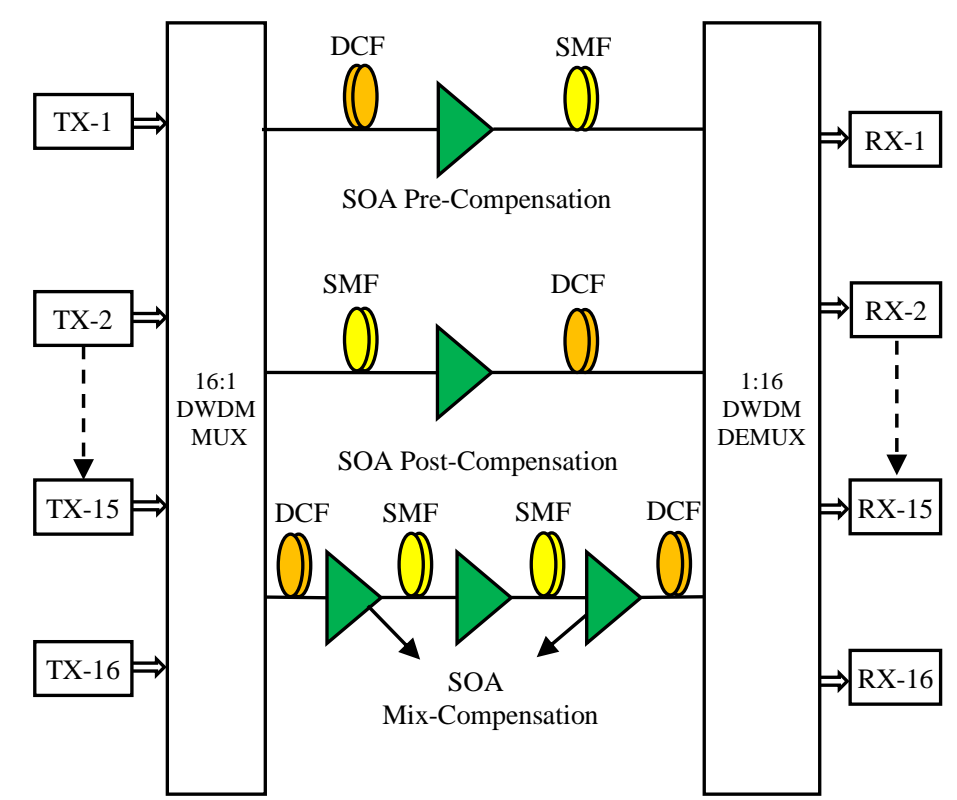

Fig.9. Basic principal of 16-channels SOA as pre-, post and Inline compensation topologies for proposed systems

Sixteen CW laser sources are used for generating different wavelength optical signals and spacing between channels is kept to $0.8 \mathrm{~nm}(100 \mathrm{GHz})$. The peak power of each Continuous Wave (CW) laser is chosen to be at $13 \mathrm{~mW}$. These optical input signals are combining through DWDM multiplexer (DWDM MUX) and transmitted over a single optical fiber consisting of SMF, DCF and SOA, this section is known as channel/media. The length of SMF and DCF are $70 \mathrm{~km}, 14 \mathrm{~km}$, respectively. Therefore, total transmission length of channel is $84 \mathrm{~km}$ with differential group delay, polarization mode dispersion (PMD) coefficient are $0.2 \mathrm{ps} / \mathrm{km}$ and $0.5 \mathrm{ps} / \mathrm{sqrt}(\mathrm{km})$, respectively. Further, the relative arrangement of SOAs, DCF and SMF are preferred according to semiconductor amplifier topologies (pre, post (boost) and in-line) and DCF is also introduced to compensate the losses in transmitted signals as shown in Fig.9. We have used an In-line amplifier topology, in which the amplifier is to be placed between two fibers (either SMF or DCF).

The wideband travelling wave SOA amplifier is introduced in an optical channel with an injection current of $0.13 \mathrm{~A}$. The power and frequency of pump laser for SOA is to be select on $0.001 \mathrm{w}$, and $193.5 \mathrm{THz}$, respectively and working at wavelength range of $1550 \mathrm{~nm}$. The optimized parameters of SOA are current injection efficiency $(\eta): 1.3$, cavity length $(\mathrm{L}): 0.6 \mathrm{~mm}$, cavity width $(\mathrm{w})$ : $0.4 \mu \mathrm{m}$, and cavity height $(\mathrm{h}): 0.4 \mu \mathrm{m}$. Furthermore, the receiver section consists of DWDM de-multiplexer (DWDM DEMUX) in which the optical signals splits into sixteen different channels/terminals. The outputs of DMUX are given to avalanche photo-detector (APD) having an ionization ratio of 0.9 and shot noise distribution select on Gaussian profile and pass through low pass electrical Bessel filter. Further, data pulse is provided to 3Rregenerator circuit where reshaping, retiming, and reamplification are to be accomplished. In last the Eye/BER analyzer is used for measuring the BER, Q-factor and eyediagrams. The all component parameters related to SMF, SOAs and DCF of the proposed DWDM optical transmission system are given in Table.1.
Table.1. Components and their optimized parameter values of simulation model

\begin{tabular}{|c|c|c|}
\hline $\begin{array}{l}\text { S. } \\
\text { No. }\end{array}$ & Components & Parameters of Eight Channels \\
\hline 1 & Data source & $\begin{array}{l}\text { Data rate: } 10 \mathrm{Gbps} \\
\text { Sample/bit: } 256 \\
\text { Bit sequence length: } 10\end{array}$ \\
\hline 2 & Pulse Generator & NRZ \\
\hline 3 & CW Laser & $\begin{array}{l}\text { Peak power: } 13 \mathrm{~mW} \\
\text { Output power: } 11.13 \mathrm{dBm} \\
\text { Channel central frequencies: } \\
193.1 \text { to } 194.6 \mathrm{THz} \\
\text { Channel spacing: } 0.8 \mathrm{~nm} \\
(100 \mathrm{GHz})\end{array}$ \\
\hline 4 & $\begin{array}{l}\text { Mach-Zehnder } \\
\text { Modulator (MZM) }\end{array}$ & $\begin{array}{l}\text { Extinction ratio: } 30 \mathrm{~dB} \\
\text { Insertion Loss: } 5 \mathrm{~dB} \\
\text { Chirp factor: } 0.5\end{array}$ \\
\hline 5 & $\mathrm{DCF}$ & $\begin{array}{l}\text { Length: } 14 \mathrm{~km} \\
\text { Attenuation Loss: } 0.33 \mathrm{~dB} / \mathrm{km} \\
\text { Dispersion: }-80 \mathrm{ps} / \mathrm{nm} . \mathrm{km} \\
\text { Dispersion slop: } 0.075 \mathrm{ps} / \mathrm{nm}^{2 / \mathrm{k}} \\
\text { Differential Group delay: } \\
0.2 \mathrm{ps} / \mathrm{km} \\
\text { Core effective area: } 80 \mu \mathrm{m}^{2} \\
\text { PMD: } 0.5 \mathrm{ps} / \mathrm{sqrt}(\mathrm{km})\end{array}$ \\
\hline 6 & SMF & $\begin{array}{l}\text { Length: } 70 \mathrm{~km} \\
\text { Attenuation Loss: } 0.2 \mathrm{~dB} / \mathrm{km} \\
\text { Dispersion: } 16 \mathrm{ps} / \mathrm{nm} . \mathrm{km} \\
\text { Dispersion slop: } 0.075 \mathrm{ps} / \mathrm{nm}^{2 / \mathrm{k}} \\
\text { Differential Group delay: } \\
0.2 \mathrm{ps} / \mathrm{km} \\
\text { Core effective area: } 80 \mu \mathrm{m}^{2} \\
\text { PMD: } 0.5 \mathrm{ps} / \mathrm{sqrt}(\mathrm{km})\end{array}$ \\
\hline 7 & SOA & $\begin{array}{l}\text { Injection current: } 0.13 \mathrm{~A} \\
\text { Pump Power: } 0.001 \mathrm{~W} \\
\text { Pump Frequency: } 193.5 \mathrm{THz} \\
\text { Wavelength: } 1550 \mathrm{~nm} \\
\text { Cavity length: } 0.6 \mathrm{~mm} \\
\text { Cavity width: } 0.4 \mu \mathrm{m} \\
\text { Cavity height: } 0.4 \mu \mathrm{m} \\
\text { current injection efficiency: } 1.3 \\
\text { Refractive Index: } 3.22 \\
\text { Gain constant: } 2.78 \mathrm{e}^{-020} \mathrm{~m}^{2} \\
\text { Carrier constant: } 1.40 \mathrm{e}^{-024} \mathrm{~m}^{-3}\end{array}$ \\
\hline 8 & Receiver & $\begin{array}{l}\text { Photo-detector: APD Diode } \\
\text { Sensitivity: }-100 \mathrm{dBm} \\
\text { Error probability: } 10^{-9}\end{array}$ \\
\hline 9 & $\begin{array}{l}\text { Low Pass Bessel } \\
\text { Filter }\end{array}$ & $-3 \mathrm{~dB}$ Bandwidth: $10 \mathrm{GHz}$ \\
\hline
\end{tabular}

\section{RESULTS AND DISCUSSION}

Prior to discussion on results, it is necessary to point out here that our main focus is to analyze the performance of semiconductor 
optical amplifiers for $16 \times 10$ Gbps DWDM optical transmission systems using pre, post or booster and In-line amplifier compensation topologies. To investigate the performance of the proposed systems, the length of optical fiber (SMF), input power, attenuation coefficient and SOAs parameters are optimized in comprehensive to pact with optimized subsequent parameters value as given in Table1. The proposed model is simulated on Optisystem7.0 simulator. Therefore, all simulations are completed using pre, post and In-line semiconductor optical amplifier compensation topologies with NRZ line coder. The quality factor (Q-factor) and bit error rate (BER) are measured by Eye/BER analyzer. Hypothetically, $\mathrm{Q}>10^{6}$ and $\mathrm{BER} \leq 10^{-9}$ are acceptable values for improved optical systems.

In order to investigate the performance of $10 \mathrm{Gbps}$ per channel transmission speed for $84 \mathrm{~km}$ optical link with satisfactory standards of Q-factor and BER values are obtained using different SOAs compensation topologies corresponding to each channel frequencies as given in Table.2. Further, the effects of different amplifiers compensation topologies on Eye-diagrams of received signals are shown in Fig.10. It can be observed that, the eye opening is much clear for In-line compensation scheme due to quality of received signal. It can also be observed from Table.2, the maximum Q-factor and minimum BER are obtained through In-line SOA compensation as compared to pre- as well as post SOAs compensation topologies.

Table.2. Q-Factor and BER of simulated model at each channel frequency using pre-, booster and In-line amplifier topology

\begin{tabular}{|c|c|c|c|c|c|c|}
\hline \multirow{2}{*}{\begin{tabular}{c}
$*$ \\
\cline { 2 - 7 }
\end{tabular}} & \multicolumn{5}{|c|}{ Semiconductor optical amplifier topology } \\
\cline { 2 - 7 } & \multicolumn{2}{|c|}{ Pre-Amplifier } & \multicolumn{2}{c|}{$\begin{array}{c}\text { In-Line } \\
\text { Amplifier }\end{array}$} & \multicolumn{2}{c|}{ Booster Amplifier } \\
\hline 193.1 & 6.695 & $9.78 \mathrm{e}^{-12}$ & 11.630 & $1.21 \mathrm{e}^{-31}$ & 4.661 & $1.42 \mathrm{e}^{-6}$ \\
\hline 193.2 & 7.496 & $3.17 \mathrm{e}^{-14}$ & 7.874 & $1.44 \mathrm{e}^{-15}$ & 4.682 & $1.40 \mathrm{e}^{-6}$ \\
\hline 193.3 & 8.735 & $9.91 \mathrm{e}^{-19}$ & 9.912 & $1.50 \mathrm{e}^{-23}$ & 4.555 & $2.59 \mathrm{e}-006$ \\
\hline 193.4 & 7.709 & $5.78 \mathrm{e}^{-15}$ & 6.487 & $3.82 \mathrm{e}^{-11}$ & 4.268 & $9.78 \mathrm{e}-006$ \\
\hline 193.5 & 5.006 & $2.54 \mathrm{e}^{-07}$ & 6.226 & $1.97 \mathrm{e}^{-10}$ & 3.062 & 0.00109261 \\
\hline 193.6 & 6.809 & $4.24 \mathrm{e}^{-12}$ & 6.261 & $1.54 \mathrm{e}^{-10}$ & 2.536 & 0.00559601 \\
\hline 193.7 & 7.694 & $5.84 \mathrm{e}^{-15}$ & 6.702 & $8.59 \mathrm{e}^{-12}$ & 2.429 & 0.00752976 \\
\hline 193.8 & 6.863 & $2.88 \mathrm{e}^{-12}$ & 7.914 & $1.05 \mathrm{e}^{-15}$ & 0 & 1 \\
\hline 193.9 & 5.058 & $1.73 \mathrm{e}^{-07}$ & 6.197 & $2.44 \mathrm{e}^{-10}$ & 2.355 & 0.00906327 \\
\hline 194 & 4.223 & $9.64 \mathrm{e}^{-06}$ & 6.034 & $6.03 \mathrm{e}^{-10}$ & 0 & 1 \\
\hline 194.1 & 3.561 & 0.00018362 & 7.888 & $1.31 \mathrm{e}^{-15}$ & 0 & 1 \\
\hline 194.2 & 3.547 & 0.000191107 & 6.698 & $8.54 \mathrm{e}^{-12}$ & 0 & 1 \\
\hline 194.3 & 2.881 & 0.00135037 & 7.570 & $1.65 \mathrm{e}^{-14}$ & 1.976 & 0.024037 \\
\hline 194.4 & 2.8335 & 0.0013707 & 7.423 & $4.85 \mathrm{e}^{-14}$ & 0 & 1 \\
\hline 194.5 & 2.824 & 0.00153242 & 7.996 & $5.90 \mathrm{e}^{-16}$ & 0 & 1 \\
\hline 194.6 & 3.006 & 0.000820179 & 7.990 & $5.75 \mathrm{e}^{-16}$ & 2.074 & 0.0147694 \\
\hline
\end{tabular}

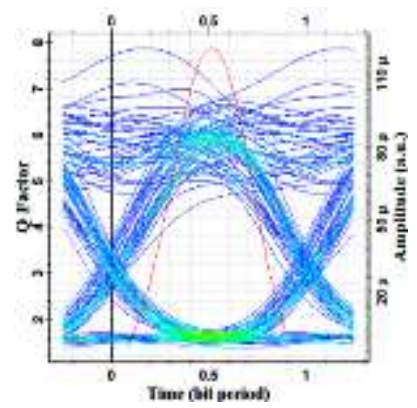

(a)

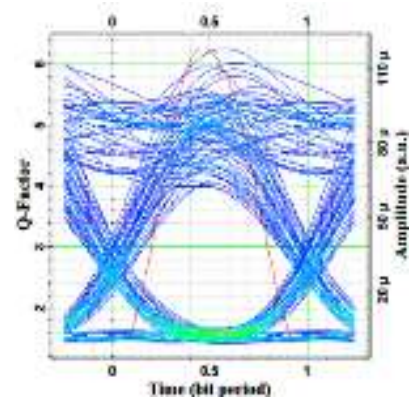

(c)

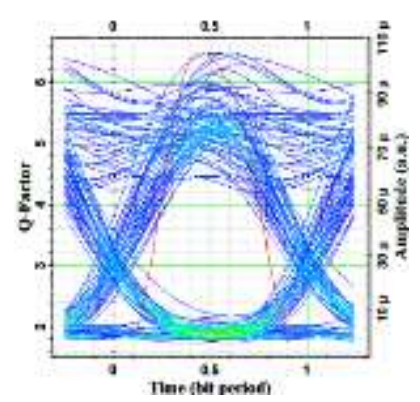

(e)

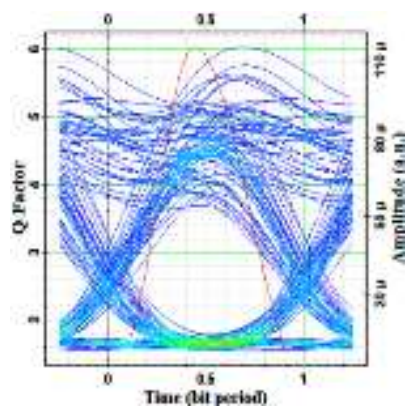

(g)

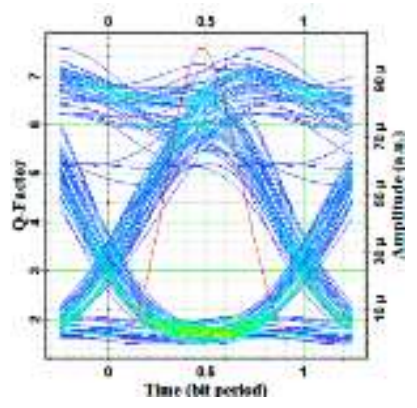

(i)

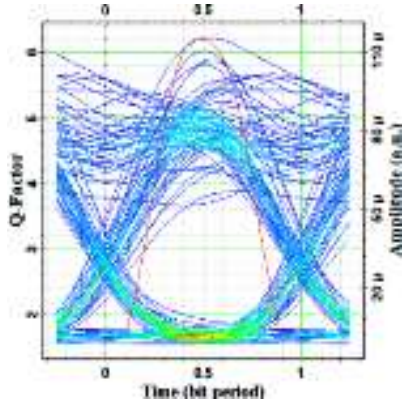

(b)

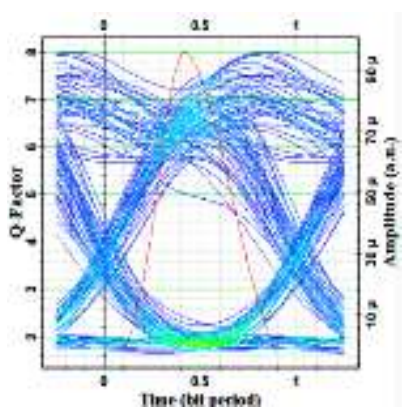

(d)

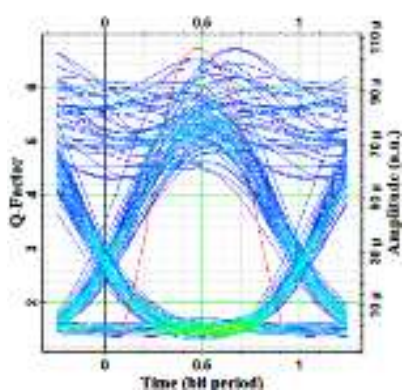

(f)

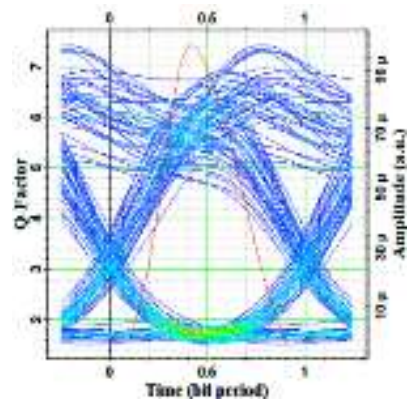

(h)

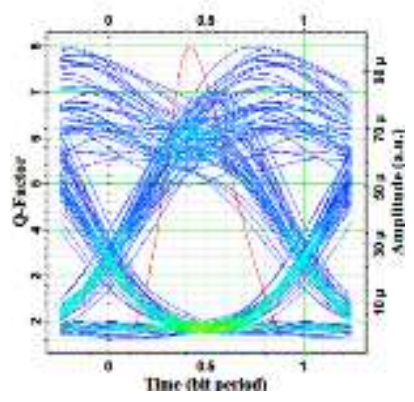

(j) 


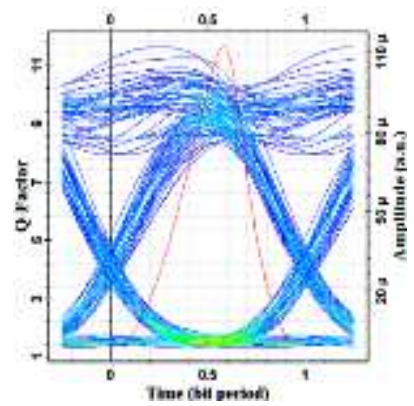

(k)

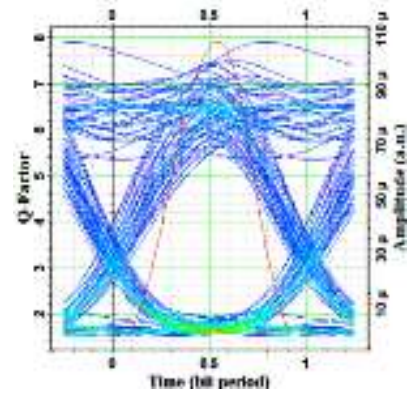

(m)

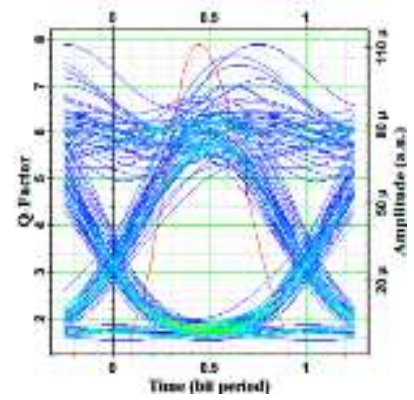

(o)

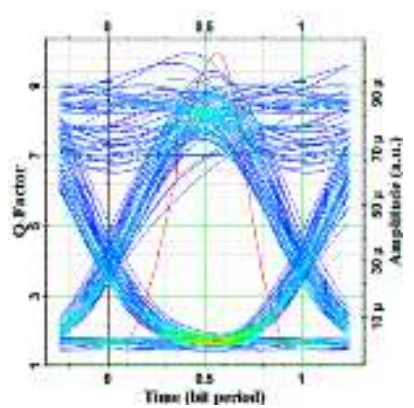

(1)

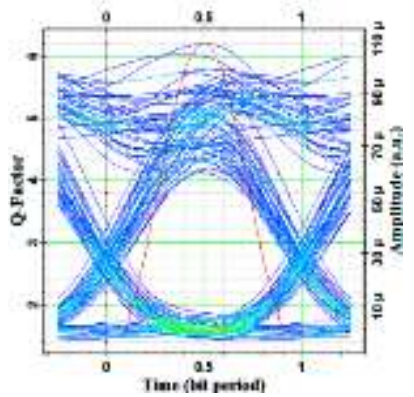

(n)

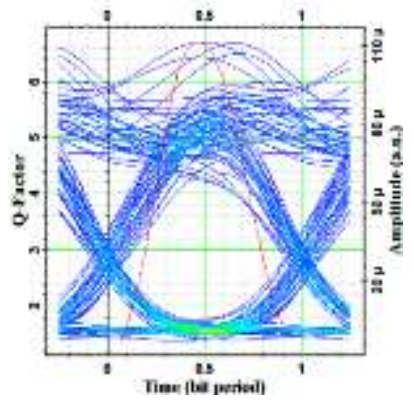

(p)
Fig.10. Eye-diagrams of received signal from proposed optical transmission system: (a)-(e) pre- SOA compensation; (f)-(j) post SOA compensation; (k)-(q) In-line SOA compensation

The Q-factor vs. frequency plot variations for pre-, booster and In-line semiconductor optical amplifiers are shown in Fig.11. The Q-factor varies from $2.8-8.7 \mathrm{~dB}, 0-4.6,6-11.6 \mathrm{~dB}$ for pre-, booster and In-line SOA amplifiers, respectively at different channel frequencies as can be observed in Fig.11. The better Qfactor is provided by In-line SOA amplifier. Similarly, the variations of BER as a function of frequency for different SOA amplifiers are shown in Fig.12. The BER for $1^{\text {st }}$ channel in the network are $9.78 \times 10^{-12}$ for pre-SOA, $1.42 \times 10^{-006}$ for booster SOA and $1.21 \times 10^{-31}$ for In-line amplifier, and further on. The least BER is provided by In-line SOA amplifier. Therefore, the proposed model offers reduced signal distortion using In-line semiconductor optical amplifier.

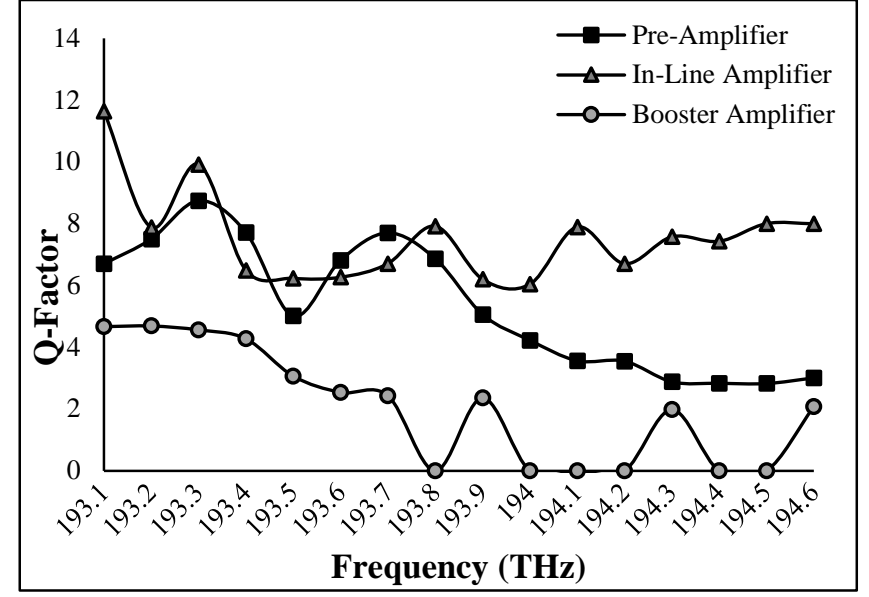

Fig.11. Q-factor vs. frequency plot variations for pre-, booster and In-line SOA amplifier

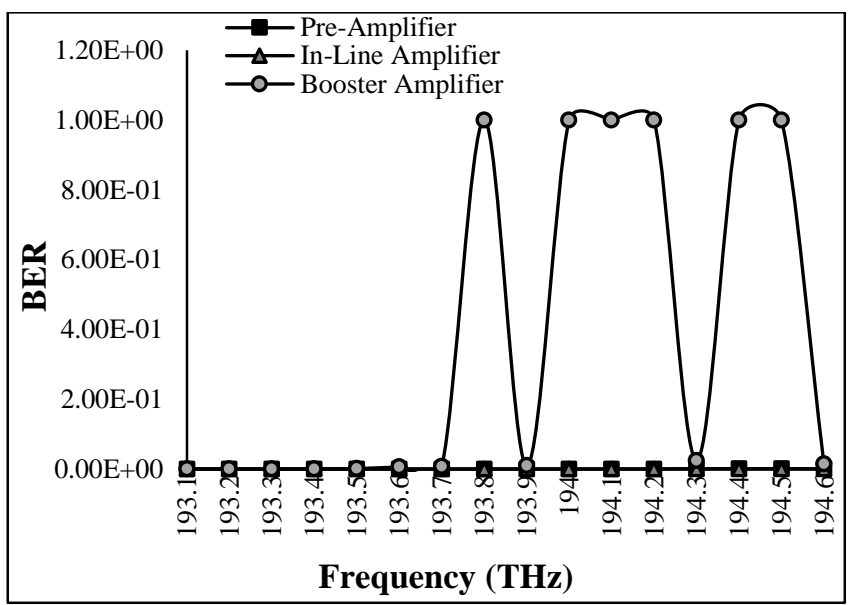

Fig.12. BER vs. frequency plot variations for pre-, booster and In-line SOA amplifier

Table.3. Performance parameters of simulated model by varying input power

\begin{tabular}{|c|c|c|c|c|c|c|c|c|}
\hline \multirow{2}{*}{$\begin{array}{c}\text { No. of } \\
\text { Channel }\end{array}$} & \multicolumn{2}{|c|}{ Power $=10 \mathrm{mw}$} & \multicolumn{2}{|c|}{$\begin{array}{c}\text { Power }= \\
12 \mathrm{mw}\end{array}$} & \multicolumn{2}{|c|}{$\begin{array}{c}\text { Power = } \\
13 \mathrm{mw}\end{array}$} & \multicolumn{2}{|c|}{$\begin{array}{c}\text { Power = } \\
14 \mathrm{mw}\end{array}$} \\
\hline & $\begin{array}{c}\text { Q- } \\
\text { factor }\end{array}$ & BER & $\begin{array}{c}\text { Q- } \\
\text { factor }\end{array}$ & BER & $\begin{array}{c}\text { Q- } \\
\text { factor }\end{array}$ & BER & $\begin{array}{c}\text { Q- } \\
\text { factor }\end{array}$ & BER \\
\hline 1 & 11.586 & $\begin{array}{c}2.009 \mathrm{e}^{-} \\
031\end{array}$ & 11.205 & $\begin{array}{c}1.602 \mathrm{e}^{-} \\
029\end{array}$ & 11.630 & $\underset{031}{1.210 \mathrm{e}^{-}}$ & 11.455 & $\underset{031}{9.197 \mathrm{e}^{-}}$ \\
\hline 2 & 8.210 & $\begin{array}{c}9.328 \mathrm{e}^{-} \\
017\end{array}$ & 7.882 & $\begin{array}{c}1.353 \mathrm{e}^{-} \\
015\end{array}$ & 7.870 & $\begin{array}{c}1.440 \mathrm{e}^{-} \\
015\end{array}$ & 8.050 & $\begin{array}{c}3.472 \mathrm{e}^{-} \\
016\end{array}$ \\
\hline 3 & 9.855 & $\begin{array}{c}2.779 \mathrm{e}^{-} \\
023\end{array}$ & 9.802 & $\underset{023}{4.672 \mathrm{e}^{-}}$ & 9.910 & $\underset{023}{1.500 \mathrm{e}^{-}}$ & 9.925 & $\begin{array}{c}1.378 \mathrm{e}^{-} \\
023\end{array}$ \\
\hline 4 & 6.616 & $\begin{array}{c}1.615 \mathrm{e}^{-} \\
011\end{array}$ & 6.723 & $\begin{array}{c}7.929 \mathrm{e}^{-} \\
012\end{array}$ & 6.487 & $\begin{array}{c}3.828 \mathrm{e}^{-} \\
011\end{array}$ & 6.549 & $\begin{array}{c}2.549 \mathrm{e}^{-} \\
011\end{array}$ \\
\hline 5 & 6.255 & $\begin{array}{c}1.635 \mathrm{e}^{-} \\
010\end{array}$ & 6.186 & $\begin{array}{c}2.561 \mathrm{e}^{-} \\
010\end{array}$ & 6.220 & $\begin{array}{c}1.970 \mathrm{e}^{-} \\
010\end{array}$ & 6.242 & $\begin{array}{c}1.778 \mathrm{e}^{-} \\
010\end{array}$ \\
\hline 6 & 6.091 & $\begin{array}{c}4.549 \mathrm{e}^{-} \\
010\end{array}$ & 6.244 & $\begin{array}{c}1.729 \mathrm{e}^{-} \\
010\end{array}$ & 6.260 & $\begin{array}{c}1.546 \mathrm{e}^{-} \\
010\end{array}$ & 6.212 & $\underset{010}{2.117 \mathrm{e}^{-}}$ \\
\hline 7 & 6.643 & $\begin{array}{c}1.277 \mathrm{e}^{-} \\
011\end{array}$ & 6.553 & $\begin{array}{c}2.356 \mathrm{e}^{-} \\
011\end{array}$ & 6.700 & $\begin{array}{c}8.590 \mathrm{e}^{-} \\
012\end{array}$ & 6.623 & $\begin{array}{c}1.465 \mathrm{e}^{-} \\
011\end{array}$ \\
\hline 8 & 7.873 & $\begin{array}{c}1.472 \mathrm{e}^{-} \\
015\end{array}$ & 7.853 & $\begin{array}{c}1.694 \mathrm{e}^{-} \\
015\end{array}$ & 7.910 & $\begin{array}{c}1.050 \mathrm{e}^{-} \\
015\end{array}$ & 7.884 & $\begin{array}{c}1.327 \mathrm{e}^{-} \\
015\end{array}$ \\
\hline
\end{tabular}




\begin{tabular}{|c|c|c|c|c|c|c|c|c|}
\hline 9 & 6.125 & $\begin{array}{c}3.811 \mathrm{e}^{-} \\
010\end{array}$ & 6.291 & $\begin{array}{c}1.341 \mathrm{e}^{-} \\
010\end{array}$ & 6.197 & $\begin{array}{c}2.446 \mathrm{e}^{-} \\
010\end{array}$ & 6.334 & $\begin{array}{c}1.007 \mathrm{e}^{-} \\
010\end{array}$ \\
\hline 10 & 6.239 & $\begin{array}{c}1.747 \mathrm{e}^{-} \\
010\end{array}$ & 6.135 & $\begin{array}{c}3.374 \mathrm{e}^{-} \\
010\end{array}$ & 6.034 & $\begin{array}{c}6.332 \mathrm{e}^{-} \\
010\end{array}$ & 6.045 & $\begin{array}{c}5.903 \mathrm{e}^{-} \\
010\end{array}$ \\
\hline 11 & 7.724 & $\begin{array}{c}4.844 \mathrm{e}^{-} \\
015\end{array}$ & 7.835 & $\begin{array}{c}2.034 \mathrm{e}^{-} \\
015\end{array}$ & 7.888 & $\begin{array}{c}1.316 \mathrm{e}^{-} \\
015\end{array}$ & 7.774 & $\begin{array}{c}3.294 \mathrm{e}^{-} \\
015\end{array}$ \\
\hline 12 & 6.576 & $\begin{array}{c}1.947 \mathrm{e}^{-} \\
011\end{array}$ & 6.869 & $\begin{array}{c}2.626 \mathrm{e}^{-} \\
012\end{array}$ & 6.698 & $\begin{array}{c}8.549 \mathrm{e}^{-} \\
012\end{array}$ & 6.565 & $\begin{array}{c}2.098 \mathrm{e}^{-} \\
011\end{array}$ \\
\hline 13 & 7.427 & $\begin{array}{c}4.922 \mathrm{e}^{-} \\
014\end{array}$ & 7.421 & $\begin{array}{c}5.139 \mathrm{e}^{-} \\
014\end{array}$ & 7.570 & $\begin{array}{c}1.655 \mathrm{e}^{-} \\
014\end{array}$ & 7.469 & $\begin{array}{c}3.569 \mathrm{e}^{-} \\
014\end{array}$ \\
\hline 15 & 7.396 & $\begin{array}{c}5.971 \mathrm{e}^{-} \\
014\end{array}$ & 7.499 & $\begin{array}{c}2.733 \mathrm{e}^{-} \\
014\end{array}$ & 7.423 & $\begin{array}{c}4.852 \mathrm{e}^{-} \\
014\end{array}$ & 7.550 & $\begin{array}{c}1.855 \mathrm{e}^{-} \\
014\end{array}$ \\
\hline 16 & 7.665 & $\begin{array}{c}3.748 \mathrm{e}^{-} \\
015\end{array}$ & 7.864 & $\begin{array}{c}1.695 \mathrm{e}^{-} \\
015\end{array}$ & 7.996 & $\begin{array}{c}5.902 \mathrm{e}^{-} \\
016\end{array}$ & 8.135 & $\begin{array}{c}1.899 \mathrm{e}^{-} \\
016\end{array}$ \\
\hline 015 & 7.905 & $\begin{array}{c}1.139 \mathrm{e}^{-} \\
015\end{array}$ & 7.990 & $\begin{array}{c}5.757 \mathrm{e}^{-} \\
016\end{array}$ & 7.978 & $\begin{array}{c}6.330 \mathrm{e}^{-} \\
016\end{array}$ \\
\hline
\end{tabular}

Furthermore, performance of the proposed optical system is optimized with design parameters and investigated in terms of QFactor and BER by varying input powers with their related graph are also depicted.

\subsection{EFFECT ON Q-FACTOR BY VARYING INPUT POWER}

Initially, the Q-factor performance of proposed optical transmission system is investigated by varying the input power with an In-line semiconductor optical amplifier (SOA). The Qfactor vs. frequency plot is evaluated from simulated model at different values of input power as shown in Fig.13. It can be observed that, the maximum Q-factor is obtained by first channel which is $11.6 \mathrm{~dB}$, after that it is varies between $10-6 \mathrm{~dB}$ according to corresponding channel frequencies. Further, when the proposed model is simulated on $10 \mathrm{~mW}, 12 \mathrm{~mW}, 13 \mathrm{~mW}$ and $14 \mathrm{~mW}$ input powers, the clear opening of eye-diagrams and maximum Qfactors are obtained at $13 \mathrm{~mW}$ input power on each channel frequency as can be seen in Fig.10. Therefore, we choose an optimized input power of CW laser at $13 \mathrm{~mW}$. Further, the output readings of optimized performance parameters with corresponding channel frequencies are given in Table.3.

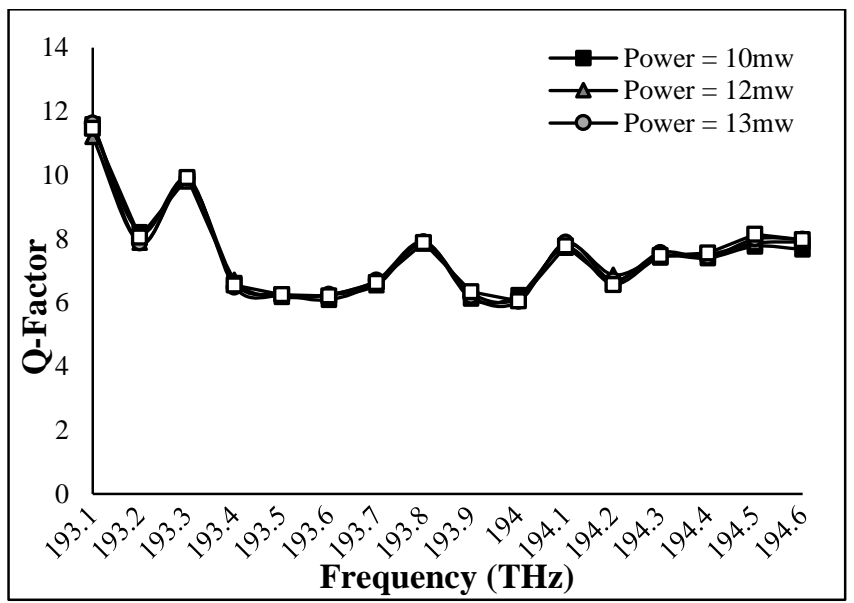

Fig.13. Variation in Q-factor vs. frequency at different input power

\subsection{EFFECT ON BER BY VARYING INPUT POWER}

Secondly, the BER performance of proposed system is investigated by varying the input power with an In-line SOA topology. The variations in BER against frequency at different input power are shown in Fig.14. It can be observed that, the minimum BER is obtained by first channel which is $1.210 \times 10^{-31}$, after that it is varies between $8.590 \times 10^{-12}$ to $1.500 \times 10^{-23}$ according to corresponding channel frequencies. The least BER is obtained at $13 \mathrm{~mW}$ power. Therefore, we choose the optimized input power of CW laser at $13 \mathrm{~mW}$. Further, the output readings of optimized parameters with corresponding input power are highlighted in Table.3.

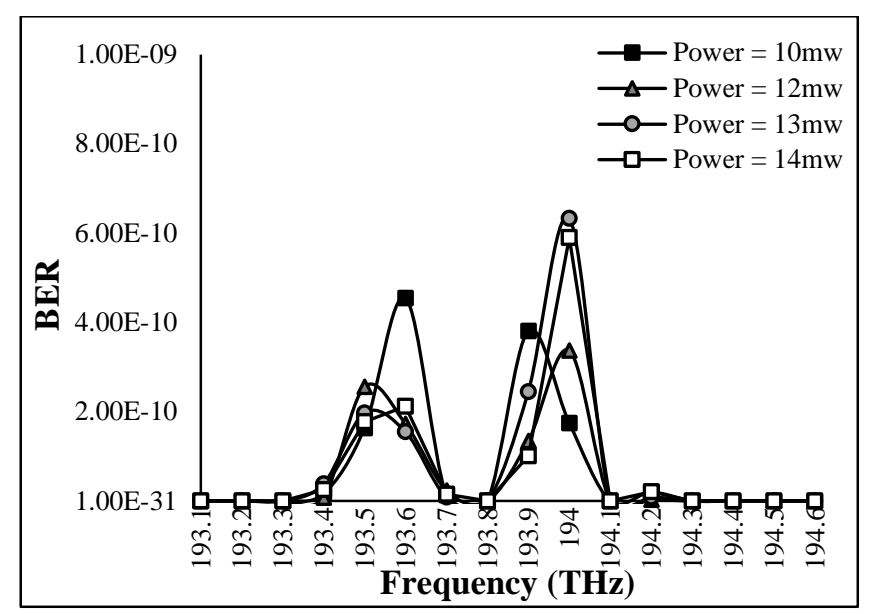

Fig.14. Variation in BER vs. frequency at different input power

From the above discussions, it is observed that, the maximum Q-factor, least BER and clear eye opening is provided by In-line SOA compensation compared to pre- as well as booster SOAs techniques. Therefore, the proposed system offers reduced signal attenuation by the In-line amplifier technique. Furthermore, overall comparative analysis of the proposed systems is compared, based on the quality of Eye-diagram, least BER and maximum Q-factor as given in Table.4.

Table.4. Comparative analysis of proposed work with similar research work reported previously

\begin{tabular}{|l|c|c|c|c|c|}
\hline \multirow{2}{*}{$\begin{array}{c}\text { Technique/ } \\
\text { Parameters }\end{array}$} & $\begin{array}{c}\text { Proposed } \\
\text { Work }\end{array}$ & {$[5]$} & {$[\mathbf{9}]$} & {$[\mathbf{1 3}]$} & {$[\mathbf{1 4}]$} \\
\cline { 2 - 6 } & In-line-SOA & EDFA & SOA+EDFA & SOA & SOA \\
\hline $\begin{array}{l}\text { Max Q-Factor } \\
\text { (dB) }\end{array}$ & 11.630 & 12.7412 & 25.91 & 10.346 & 8.2712 \\
\hline Min BER & $\begin{array}{c}1.210 \\
\mathrm{e}^{-031}\end{array}$ & $\begin{array}{c}1.63383 \\
\mathrm{e}^{-037}\end{array}$ & $\begin{array}{c}1.010 \\
\mathrm{e}^{-040}\end{array}$ & $\begin{array}{c}4.844 \\
\mathrm{e}^{-015}\end{array}$ & $\begin{array}{c}6.328 \\
\mathrm{e}^{-010}\end{array}$ \\
\hline Gain (dB) & - & 5.2840 & 6.9470 & 36.5230 & 30.1430 \\
\hline $\begin{array}{l}\text { Output Power } \\
\text { (dBm) }\end{array}$ & 11.13 & 13.450 & 13.80 & 21.36 & 19.20 \\
\hline $\begin{array}{l}\text { SMF Length } \\
(\mathrm{km})\end{array}$ & 70 & 150 & 100 & 60 & 40 \\
\hline $\begin{array}{l}\text { DCF/CFBG } \\
\text { Length } \\
(\mathrm{km} / \mathrm{mm})\end{array}$ & $14 \mathrm{~km}$ & $45 \mathrm{~mm}$ & $16 \mathrm{~km}$ & $20 \mathrm{~km}$ & $15 \mathrm{~km}$ \\
\hline Cost & Medium & Low & Medium & High & Medium \\
\hline
\end{tabular}




\section{CONCLUSIONS}

The present work highlighted the performance of designed $16 \times 10 \mathrm{Gbps}$ DWDM optical transmission system using different semiconductor optical amplifiers (pre-SOA, booster-SOA and Inline-SOA). The performance parameters of designed $84 \mathrm{~km}$ optical link is investigated on each channel in terms of eyediagrams, bit error rate (BER) and Q-factor by varying input power $(\mathrm{mW})$. Further, we evaluate the comparative performance of each channel for SOA topologies with cost analysis and it is found that the optimized In-line SOA amplifier topology gives the best performance (better Q-factor and least BER) compared to pre- as well as booster amplifiers. In future, the proposed techniques can be applied to a complex optical transmission system with large number of channels to compensate the attenuation complications.

\section{REFERENCES}

[1] S. Parkash, A. Sharma, H. Singh and H.P. Singh, "Performance Investigation of 40Gb/s DWDM Over Free Space Optical Communication System using RZ Modulation Format", Advances in Optical Technologies, Vol. 2016, pp. 1-8, 2016.

[2] V. Bobrovs, Alsevska, S. Olonkins, L. Gegere and G. Lvanovs, "Comparative performance of Raman-SOA and Raman-EDFA Hybrid Optical Amplifiers in DWDM Transmission Systems", International Journal of Physical Sciences, Vol. 8, No. 39, pp. 1898-1906, 2013.

[3] A.H. Gnauck, L.D. Garrett, Y. Danziger, U. Levy and M. Tur, "Dispersion and Dispersion-Slope Compensation of NZDSF Over the Entire C Band using Higher-Order-Mode Fibre”, Electronics Letters, Vol. 36, No. 23, pp. 1946-1947, 2000.

[4] M. Sumetsky and B.J. Eggleton, "Fiber Bragg Gratings for Dispersion Compensation in optical Communication Systems", Proceedings of International Conference on Ultrahigh-Speed Optical Transmission Technology, pp. 277-299, 2005.

[5] M.L. Meena and Raj Kumar Gupta, "Design and Comparative Performance Evaluation of Chirped FBG Dispersion Compensation with DCF Technique for DWDM Optical Transmission Systems", Optik, Vol. 188, pp. 212224, 2019.

[6] G.P. Agrawal, "Fiber-Optic Communication Systems", $3^{\text {rd }}$ Edition, John Wiley and Sons, 2007.
[7] R. Srivastava and Y.N. Singh, "Fiber Optic Loop Buffer Switch Incorporating 3R Regeneration", Optical and Quantum Electronics, Vol. 42, No. 5, pp. 297-311, 2011.

[8] S. Singh, R. Randhawa and R.S. Kaler, "Handbook on Optical Amplifiers", Lambert Academic Publisher, 2015.

[9] Deepak Malik, Kuldip Pahwa and Amit Wason, "Performance Optimization of SOA, EDFA, Raman and hybrid Optical Amplifiers in WDM Network with Reduced Channel Spacing of 50GHz", Optik, Vol. 127, pp. 1113111137, 2016.

[10] M.N. Islam, "Raman Amplifiers for Telecommunications 2: Sub-Systems and Systems", Springer,2004.

[11] F.M. Mustafa, A.A.M. Khalaf and F.A. Elgeldawy, "MultiPumped Raman Amplifier for Long-Haul UW-WDM Optical Communication Systems: Gain Flatness and Bandwidth Enhancements", Proceedings of $15^{\text {th }}$ International Conference on Advanced Communication Technology, pp. 122-127, 2013.

[12] M.J. Connely, "Semiconductor Optical Amplifiers", Kluwer Academic Publishers, 2004.

[13] S. Singh and R.S. Kaler, "Hybrid Optical Amplifiers for $64 \times 10$ Gbps Dense Wavelength Division Multiplexed System”, Optik, Vol. 124, pp. 1311-1313, 2013.

[14] J. Helina Rajini and S. Tamil Selvi, "Performance Analysis of Hybrid Optical Amplifier for $64 \times 10$ Gbps DWDM Systems", Asian Journal of Applied Sciences, Vol. 8, No. 1, pp. 46-54, 2015.

[15] S. Singh, R.S. Kaler and A. Singh, "Performance Evaluation of EDFA, RAMAN and SOA Optical Amplifier for WDM Systems", Optik, Vol. 124, pp. 95-101, 2013.

[16] Ramandeep Kaur, Rajneesh Randhawa and R.S. Kaler, "Performance Evaluation of Optical Amplifier for $16 \times 10$, $32 \times 10$ and $64 \times 10$ Gbps WDM System", Optik, Vol. 124, pp. 693-700, 2013.

[17] S. Singh and R.S. Kaler, "Wide-Band Optical Wavelength Converter Based on Four-Wave Mixing using Optimized Semiconductor Optical Amplifier", Fiber and Integrated Optics, Vol. 25, No. 3, pp. 213-230, 2006.

[18] S. Singh and R.S. Kaler, "Review on Recent Developments in Hybrid Optical Amplifier for Dense Wavelength Division Multiplexed System", Optical Engineering, Vol. 54, No. 10, pp. 100901-100910, 2005.

[19] Kyriakos E. Zoiros, "Special Issue on Applications of Semiconductor Optical Amplifiers", Applied Sciences, Vol. 8, No. 7, pp. 1185-1189, 2018.

[20] Nabil Elsheikh Mohamed Elmak and Amin Babiker Nabi Mustafa, "Performance Analysis of Optical Amplifiers (EDFA and SOA)", IOSR Journal of Electronics and Communication Engineering, Vol. 12, No. 2, pp. 5-7, 2017. 